\title{
Patterns of healthcare utilisation for respiratory complications of adults with neuromuscular disease: a population study
}

\author{
Louise Rose 1,2,3,4 , Douglas McKim 5,6,7, David Leasa ${ }^{8,9}$, Mika Nonoyama 10,11,12, \\ Anu Tandon ${ }^{13,14}$, Yu Qing Bai ${ }^{14,15}$, Reshma Amin ${ }^{14,16,17}$, Sherri Katz ${ }^{7,18,19}$, \\ Roger Goldstein ${ }^{14,20}$ and Andrea Gershon ${ }^{13,21,22}$
}

@ERSpublications

One-third of adults with neuromuscular disease received specialist respiratory care. Emergent healthcare use was substantial, emphasising an urgent need to improve community and social supports. http://ow.ly/FluZ30leuLk

Cite this article as: Rose L, McKim D, Leasa D, et al. Patterns of healthcare utilisation for respiratory complications of adults with neuromuscular disease: a population study. Eur Respir J 2018; 52: 1800754 [https://doi.org/10.1183/13993003.00754-2018].

ABSTRACT Our objective was to quantify health service utilisation including monitoring and treatment of respiratory complications for adults with neuromuscular disease (NMD), identifying practice variation and adherence to guideline recommendations at a population level.

We conducted a population-based longitudinal cohort study (2003-2015) of adults with NMD using hospital diagnostic and health insurance billing codes within administrative health databases.

We identified 185586 adults with NMD. Mean age 52 years, 59\% female. 41173 (22\%) went to an emergency department for respiratory complications on average 1.6 times every 3 years; 14947 (8\%) individuals were admitted to hospital 1.4 times every 3 years. Outpatient respiratory specialist visits occurred for 64084 (35\%) with four visits every 3 years, although substantial variation in visit frequency was found. 157285 (85\%) went to the emergency department (all-cause) almost 4 times every 3 years, 100052 (54\%) were admitted to hospital. Individuals with amyotrophic lateral sclerosis/motor neurone disease (ALS/MND) had more emergency department visits compared with other types of NMD ( $\mathrm{p}<0.0001)$.

One-third of adults with NMD received respiratory specialist care at a frequency recommended by professional guidelines, although substantial variation exists. Emergent healthcare utilisation was substantial, emphasising the burden of NMD on the healthcare system and urgent need to improve community and social supports, particularly for ALS/MND patients. 
Affiliations: 'Sunnybrook Health Sciences Centre and Sunnybrook Research Institute, Toronto, ON, Canada. ${ }^{2}$ Florence Nightingale Faculty of Nursing, Midwifery and Palliative Care, Kings College London, London, UK. ${ }^{3}$ Lawrence S. Bloomberg Faculty of Nursing and Faculty of Medicine, University of Toronto, Toronto, ON, Canada. ${ }^{4}$ Institute of Clinical Evaluative Sciences, Toronto, ON, Canada. ${ }^{5}$ The Ottawa Hospital Respiratory Rehabilitation and The Ottawa Hospital Sleep Centre, Ottawa, ON, Canada. ${ }^{6}$ Ottawa Hospital Research Institute, Ottawa, ON, Canada. ${ }^{7}$ Faculty of Medicine, University of Ottawa, Ottawa, ON, Canada. ${ }^{8}$ Dept of Medicine, Divisions of Critical Care and Respirology, London Health Sciences Centre, London, ON, Canada. ${ }^{9}$ Faculty of Medicine, Western University, London, ON, Canada. ${ }^{10}$ University of Ontario Institute of Technology, Toronto, ON, Canada. ${ }^{11}$ Child Health Evaluative Sciences and Respiratory Therapy, SickKids, Toronto, ON, Canada. ${ }^{12}$ Rehabilitation Sciences and Physical Therapy, University of Toronto, Toronto, ON, Canada. ${ }^{13}$ Sunnybrook Health Sciences Centre, Toronto, ON, Canada. ${ }^{14}$ Faculty of Medicine, University of Toronto, Toronto, ON, Canada. ${ }^{15}$ Institute of Health Policy, Management and Evaluation, Toronto, ON, Canada. ${ }^{16}$ Division of Respiratory Medicine, SickKids, Toronto, ON, Canada. ${ }^{17}$ SickKids Research Institute, Toronto, ON, Canada. ${ }^{18}$ Division of Respirology, Children's Hospital of Eastern Ontario, Ottawa, ON, Canada. ${ }^{19}$ Children's Hospital of Eastern Ontario Research Institute, Ottawa, ON, Canada. ${ }^{20}$ West Park Healthcare Centre, Toronto, ON, Canada. ${ }^{21}$ Sunnybrook Research Institute and Institute of Clinical Evaluative Sciences, Toronto, ON, Canada. ${ }^{22}$ Dept of Medicine, University of Toronto, Toronto, ON, Canada.

Correspondence: Louise Rose, Sunnybrook Health Sciences Centre, D108, Bayview Avenue, Toronto, ON M4N 3M5, Canada. E-mail: louise.rosedutoronto.ca

\section{Introduction}

Individuals with neuromuscular disease (NMD) experience progressive respiratory muscle weakness, and are at high risk of respiratory insufficiency [1] due to hypoventilation during sleep [2, 3], recurrent atelectasis and pneumonia [4] resulting from decreased cough effectiveness [5]. NMDs include a heterogeneous spectrum of diseases that affect muscle and nerve function, many of which are genetic, represent extremely complex pathophysiology, have varying degrees of severity and require high levels of care [6]. Although some NMDs are rare, when considered as a group these disorders are relatively common with an estimated prevalence of about 1 in 3000 [7].

For most individuals with NMD, increased healthcare requirements related to respiratory problems and the need for assistive respiratory technology indicate progression of disease and increased morbidity and mortality [8]. Acute respiratory failure due to respiratory infection is a frequent reason for unplanned hospital admission, although there are limited empirical data on patterns of healthcare utilisation in this patient population. Chronic respiratory failure is the most common cause of death [7]. The primary aim of respiratory management is prevention and timely management of respiratory-related complications, as well as symptom control [9]. Proactive monitoring of pulmonary function [8], appropriate use of respiratory interventions such as lung volume recruitment [5], manual or mechanical cough augmentation [10] and noninvasive ventilation [11] may either prevent or manage progressive respiratory insufficiency, thus reducing unplanned hospital admissions and improving health-related quality of life and longevity.

Several consensus documents inform evidence- and expert-derived best practices which, when adopted, may reduce clinical practice variation and improve the appropriateness of respiratory assessment and management of individuals with NMD [7, 9, 12-19]. Recommendations for management of respiratory health in this population include: access to respiratory and/or sleep specialists early in the disease course; measurement at baseline and over time of lung function, oximetry and as indicated carbon dioxide; and outpatient clinic visits to assess respiratory symptom progression every 3, 6 or 12 months dependent on disease progression and initiation of ventilation. Despite these consensus statements, surveys of service providers or care recipients suggest variation in respiratory management practices [20, 21], and respiratory complications remain a primary cause of morbidity and mortality [8].

To address gaps in knowledge related to adherence to guideline recommendations at a population level, we conducted a North American population-based cohort study using administrative databases to quantify health service utilisation, with a particular focus on services used for assessing, monitoring and treating respiratory complications for adults with NMD. We hypothesised that frequency of monitoring and respiratory specialist care would be variable, but should increase with initiation of mechanical ventilation. Secondary objectives were to explore: 1) all-cause publicly funded health service utilisation, 2) variables associated with emergency department presentation or hospital admission, and 3) changes in healthcare utilisation before and after initiation of home mechanical ventilation (HMV).

\section{Methods}

We conducted a retrospective longitudinal population-based cohort study from April 1, 2003 to March 31, 2015 using health administrative databases for the province of Ontario, Canada held at the Institute for Clinical Evaluative Sciences (ICES; www.ices.on.ca) using unique encoded identifiers to link databases. These databases contain anonymised data for all Ontario residents. Ontario is the most populous 
Canadian province with a population of approximately 13 million [22]. Costs of all medically necessary care are covered for all residents by universal public health insurance funded through general taxation. Respiratory equipment such as ventilators, continuous positive airway pressure (CPAP) and bilevel devices is publicly funded through the Assistive Devices Program (ADP).

\section{Data sources and population}

We linked databases at the level of the individual using unique encoded identifiers to identify: 1) hospitalisations including demographic, diagnostic and procedural data, and in-hospital death in the Discharge Abstract Database; 2) emergency department presentations in the National Ambulatory Care Reporting System; 3) physician billings including procedures in the Ontario Health Insurance Plan (OHIP) database [23]; 4) approvals of assistive devices including ventilators, suction equipment and supplies in the ADP database; 5) inpatient rehabilitation using the National Rehabilitation Reporting System; 6) facility-based continuing (residential) care services in the Continuing Care Reporting System; 7) home care services using the Home Care Database; and 8) death outside of hospital using the Registered Persons Database. To describe immigration status we used the Immigration, Refugees and Citizenship Canada's Permanent Resident Database. This database contains individual-level demographic data on all immigrants who became permanent residents between 1985 and 2012. Following well-established methods, both neighbourhood income and urban/rural place of residence were ascertained using postal codes and linking to Statistics Canada census data [24]. To identify respiratory specialist care, we used the ICES Physician Database that contains information about all physicians in Ontario.

We created a cohort of adults aged 18-105 years using hospital diagnostic codes (International Classification of Diseases (ICD)-9 and -10)) or physician billing codes a priori considered specific to NMD within health administration databases from April 1, 2003 to March 31, 2008. Within this 5-year period we sought the first instance of these codes considered the "most responsible" or secondary contributing diagnoses in the hospitalisation database [25] indicating hospital admission with a NMD diagnosis. For individuals with a NMD diagnostic code found using the ambulatory care database, we included only those whose NMD diagnosis was confirmed by looking backwards and forwards in time in the hospitalisation database (1998-2014) for a NMD-related hospital admission. For those without hospital admission, we reviewed the physician billing database for NMD-related billing in these years. For individuals identified using the OHIP billing code 349 (NMD diagnosis nonspecific), we again looked backwards and forwards in the hospitalisation (1998-2014) and ambulatory care (2000-2014) databases to establish NMD diagnosis, and sought OHIP billings from a neurologist or for an electromyogram enabling more accurate classification.

We grouped diagnostic codes for NMD (ICD and OHIP) into 12 categories: amyotrophic lateral sclerosis/ motor neurone disease (ALS/MND), cerebral palsy [26], Guillain-Barré syndrome [27], metabolic disorders, multiple sclerosis [28], muscular dystrophy, myasthenia gravis [29], neuromuscular disorders (nonspecific), neuropathy, post-polio syndrome, spina bifida and spinal muscular atrophy. For modelling purposes we further grouped these NMD categories as rapidly progressive (ALS/MND), slowly or nonprogressive (Guillain-Barré syndrome, myasthenia gravis, neuropathy, post-polio syndrome and spinal muscular atrophy), variably progressive (metabolic disorders, muscular dystrophy and neuromuscular disorders (nonspecific)) and other (cerebral palsy, multiple sclerosis and spina bifida) [30]. We used previously validated case definitions for asthma [31], chronic obstructive pulmonary disease (COPD) [32] and congestive heart failure (CHF) [33], and ICD-10 codes for cardiomyopathy and arrhythmias. We determined Collapsed Aggregated Diagnosis Groups (CADGs) [34] and calculated the Charlson Comorbidity Index score considering all hospitalisations within 2 years prior to identification of NMD.

\section{Outcomes}

To evaluate healthcare utilisation we followed our 2003-2008 cohort from identification of NMD to March 31, 2015 (minimum of 7 years). Our primary outcome was health service utilisation for assessment, monitoring and management of respiratory complications, including respiratory-related emergency department presentations and hospital admissions; consultations with a respiratory specialist (in-hospital and ambulatory/outpatient clinic); pulmonary function testing (PFT); polysomnography (PSG); access to community respiratory therapists; and use of respiratory therapies in the community, including oxygen, mechanical ventilation and airway clearance devices.

Additional healthcare utilisation outcomes included: all-cause emergency department visits, hospital and intensive care unit (ICU) admissions; duration of emergency department, hospital and ICU stay; outpatient clinic visits and inpatient services by specialists other than respiratory; inpatient rehabilitation; homecare services; and long-term care residence. We examined associations of the a priori identified demographic and clinical variables with frequency of emergency department presentation and hospital 
admission: NMD category [30]; age; sex; neighbourhood income quintile; rural residence; immigration status; ventilation status; use of other medical technologies; comorbidities (i.e. COPD, asthma, CHF, myopathy and arrhythmias); and CADGs [34]. We also compared healthcare utilisation in the 1 and 3 years before and after initiation of mechanical ventilation in a subset of the cohort with 3 years of follow-up data after initiation of mechanical ventilation.

\section{Ethical considerations}

We conducted our study according to a pre-specified protocol approved by the Research Ethics Board at Sunnybrook Health Sciences Centre (Toronto, ON, Canada) and according to privacy regulations of the ICES.

\section{Statistical analyses}

We used descriptive statistics to characterise our cohort. We report counts, overall proportion and mean $\pm \mathrm{SD}$ frequency every 3 years per individual for rates of healthcare utilisation, as well as mean \pm SD and median (interquartile range (IQR)) duration of healthcare utilisation (i.e. hospital admission). We created generalised linear regression models with negative binomial distribution to examine variables associated with frequency of emergency department presentation or hospital admissions while controlling for the aforementioned variables and using exposure time as an offset variable. We compared healthcare utilisation before and after initiation of HMV using t-tests. We conducted analyses in SAS Enterprise Guide 7.1 (SAS Institute, Cary, NC, USA).

\section{Results}

In the 5-year cohort creation period, we identified 185586 adults with NMD. Of these, we identified 2249 (1.2\%) through emergency department presentation, 10656 (5.7\%) through hospitalisation and 172681 (93.0\%) through physician billing only. At baseline, mean age was 52 years, 59\% were female and $89 \%$ lived in an urban location. Asthma (15\%) and COPD (12\%) were fairly common respiratory comorbidities (table 1; see supplementary table $\mathrm{S} 1$ for the 12 disease groupings).

\section{Health service utilisation for respiratory complications}

During fiscal years 2003-2014, subsequent to identification of NMD diagnosis, 41173 (22\%) individuals went to the emergency department for respiratory reasons for a total of 85066 visits with a median (IQR) length of stay $5.2(2.5-12.4)$ h. 14947 (8\%) individuals were admitted to hospital (24929 admissions) for respiratory reasons with a median (IQR) length of stay 7 (3-17) days. On average, individuals with NMD went to the emergency department 1.6 times every 3 years and were admitted to hospital 1.4 times every 3 years for respiratory causes. Outpatient clinic visits to a respiratory specialist occurred for 64084 (35\%) individuals with an average of four visits every 3 years, although there was substantial variation in the number of visits per individual and those with ALS/MND or muscular dystrophy had an average of 17 and 7.5 visits per individual every 3 years, respectively. In-hospital pulmonologist consultation occurred for 17489 (9\%) individuals with the mean \pm SD number of consultations falling from 6.6 \pm 13.7 in 2003-2005 to $4.5 \pm 16.1$ in 2012-2014. PFT was conducted in 60388 (33\%) individuals with the mean \pm SD number of tests every 3 years for the entire group receiving testing ranging from $1.9 \pm 2.0$ in $2003-2005$ to $2.2 \pm 3.4$ in 2012-2014, and similar frequencies for individuals with muscular dystrophy and ALS/MND (supplementary table S2). PSG was conducted in 26070 (14\%) individuals with testing frequency every 3 years similar over time (table 2; see supplementary table S2 for all health service utilisation for individuals with muscular dystrophy and ALS/MND). A publicly funded CPAP device was supplied to $7737(4.2 \%)$ individuals, long-term oxygen therapy (LTOT) to $4750(2.6 \%)$ individuals and $1446(0.8 \%)$ individuals were supplied a publicly funded ventilator (invasive (176 individuals) or noninvasive bilevel device (1270 individuals)) for HMV. Over $50 \%$ of individuals requiring LTOT had COPD and/or CHF as a comorbidity (table 3$)$. Only $71(0.04 \%)$ individuals received services from publicly funded respiratory therapy providers in the community, reflective not of a lack of need but of the lack of public funding in Ontario for community respiratory therapy.

\section{Other health service utilisation}

During fiscal years 2003-2014, subsequent to identification of NMD diagnosis, 157285 (85\%) individuals went to the emergency department for 1202800 visits (all-cause) with a median (IQR) length of stay 19.1 (7.6-47) h. 100052 (54\%) individuals were admitted to hospital (301064 admissions) with a median (IQR) length of stay 9 (3-28) days. On average, individuals with NMD visited the emergency department (all-cause) almost 4 times every 3 years and were admitted to hospital (all-cause) twice every 3 years. One or more ICU admissions were required for 29453 (16\%) individuals with a total of 53200 ICU admissions and a median (IQR) length of stay of 3.6 (1.6-8.4) days. Outpatient clinic visits to physician specialists other than respiratory specialists occurred for 184950 (99.6\%) individuals with average visit frequency every 3 years increasing over time from 23.8 to $30.1 ; 128775$ (69\%) individuals had in-hospital specialist 
TABLE 1 Cohort characteristics (2003-2008)

\begin{tabular}{|c|c|c|c|c|c|}
\hline & Total & Rapidly progressive & Slowly or nonprogressive ${ }^{\Uparrow}$ & Variably progressive ${ }^{+}$ & Other ${ }^{\S}$ \\
\hline Age years & $52 \pm 16.4$ & $71.2 \pm 11.9$ & $53.51 \pm 16.8$ & $54.39 \pm 16.1$ & $45.69 \pm 15.2$ \\
\hline $18-39$ years & 44044 (23.7) & $15(1.7)$ & 7023 (21.9) & $19640(18.7)$ & $17366(36.6)$ \\
\hline 40-64 years & $96903(52.2)$ & $185(21.4)$ & $16330(50.9)$ & 55523 (52.9) & $24865(52.0)$ \\
\hline$\geqslant 65$ years & $44639(24.1)$ & $664(76.9)$ & $8701(27.1)$ & $29702(28.3)$ & $5572(11.7)$ \\
\hline Male & 75667 (40.8) & $510(59.0)$ & $14258(44.5)$ & $45455(43.4)$ & 15444 (32.3) \\
\hline \multicolumn{6}{|c|}{ Neighbourhood income quintile $f$} \\
\hline 1 (poorest/lowest) & $35603(19.2)$ & $194(22.6)$ & 6449 (20.2) & $19547(18.7)$ & 9413 (19.8) \\
\hline 2 & 36884 (19.9) & $183(21.3)$ & $6794(21.2)$ & $20546(19.7)$ & $9361(19.6)$ \\
\hline 3 & $36488(19.7)$ & $175(20.4)$ & $6695(20.9)$ & $20324(19.4)$ & 9294 (19.5) \\
\hline 4 & $37672(20.3)$ & $153(17.8)$ & $6103(19.1)$ & $21746(20.8)$ & $9670(20.3)$ \\
\hline \multicolumn{6}{|l|}{ Immigration status ๆๆ } \\
\hline Citizen & 169504 (91.3) & 812 (94.0) & $28072(87.6)$ & 95935 (91.5) & 44685 (93.5) \\
\hline Immigrant $^{++}$ & $12917(7.0)$ & $44(5.1)$ & $3230(10.1)$ & 7107 (6.8) & 2536 (5.3) \\
\hline Refugee & $3154(1.7)$ & $8(0.9)$ & $752(2.4)$ & $1815(1.7)$ & $579(1.2)$ \\
\hline \multicolumn{6}{|l|}{ Comorbidity } \\
\hline COPD & $22162(11.9)$ & $264(30.6)$ & 4070 (12.7) & $14285(13.6)$ & $3543(7.4)$ \\
\hline Asthma & $28720(15.5)$ & $133(15.4)$ & $4769(14.9)$ & $17414(16.6)$ & $6404(13.4)$ \\
\hline $\mathrm{CHF}$ & $8310(4.5)$ & $134(15.5)$ & $1570(4.9)$ & $5606(5.4)$ & $1000(2.1)$ \\
\hline \multicolumn{6}{|c|}{ Charlson Comorbidity Index ${ }^{\S \S}$} \\
\hline 0 & 28360 (15.3) & $369(46.4)$ & $5034(57.6)$ & 14822 (53.3) & 8135 (70.5) \\
\hline 1 (score 1-3) & $16871(9.1)$ & $361(45.4)$ & 3050 (34.9) & 10502 (37.8) & $2958(25.6)$ \\
\hline 2 (score $\geqslant 4$ ) & $3642(2.0)$ & 66 (8.3) & $650(7.4)$ & $2475(8.9)$ & $451(3.9)$ \\
\hline
\end{tabular}

Data are presented as $\mathrm{n}$, mean \pm SD or $\mathrm{n}(\%)$; some percentages may not sum to 100 due to rounding. COPD: chronic obstructive pulmonary disease; CHF: congestive heart failure. \#: amyotrophic lateral sclerosis/motor neurone disease; ": Guillain-Barré syndrome, myasthenia gravis, neuropathy, post-polio syndrome and spinal muscular atrophy; ${ }^{+}$: metabolic disorders, muscular dystrophy and neuromuscular disorders (nonspecific); ${ }^{\S}$ : cerebral palsy, multiple sclerosis and spina bifida; ${ }^{f}$ : unable to establish for 533 (0.3\%) individuals; ${ }^{\# \#}$ : unable to establish for $103(0.06 \%)$ individuals; ${ }^{\text {ก1: }}$ unable to establish for $11(0.0006 \%)$ individuals; ${ }^{++}$: resident within Ontario as holding either Canadian permanent residency status or a work, student or other form of visa; ${ }^{\S \S}$ : calculated for those admitted to hospital considering all hospitalisations within 2 years prior to the index event (48873 patients).

TABLE 2 Health service utilisation after neuromuscular disease diagnosis in 185586 individuals

\begin{tabular}{|c|c|c|c|c|c|c|}
\hline & Individuals & $\begin{array}{l}\text { Overall visits per } \\
\text { individual }\end{array}$ & \multicolumn{4}{|c|}{ Visits per 3 years per individual ${ }^{\#}$} \\
\hline Respiratory-related emergency department visits & $41173(22.2)$ & $2.1 \pm 2.6 ; 1(1-2)$ & $1.5 \pm 1.2$ & $1.5 \pm 1.3$ & $1.6 \pm 1.4$ & $1.6 \pm 1.6$ \\
\hline Respiratory-related hospital admission & $14947(8.0)$ & $1.7 \pm 1.6 ; 1(1-2)$ & $1.4 \pm 1.0$ & $1.4 \pm 1.0$ & $1.4 \pm 1.0$ & $1.4 \pm 1.1$ \\
\hline Pulmonology outpatient clinic & $64084(34.5)$ & $5.9 \pm 22.4 ; 2(1-5)$ & $3.8 \pm 11.8$ & $3.9 \pm 13.1$ & $4.1 \pm 11.9$ & $4.0 \pm 11.6$ \\
\hline Hospital pulmonology consult & $17489(9.4)$ & $6.6 \pm 28.4 ; 3(1-6)$ & $6.6 \pm 13.7$ & $6.4 \pm 17.9$ & $5.0 \pm 19.6$ & $4.5 \pm 16.1$ \\
\hline Pulmonary function tests & $60388(32.5)$ & $3.2 \pm 5.4 ; 2(1-3)$ & $1.9 \pm 2$ & $2.1 \pm 2.8$ & $2.2 \pm 3.2$ & $2.2 \pm 3.4$ \\
\hline Hospital consult (nonpulmonology) & $128775(69.4)$ & $20.2 \pm 36.2 ; 6(2-22)$ & $12.2 \pm 21.5$ & $11.9 \pm 22.2$ & $11 \pm 21.3$ & $11.1 \pm 21.4$ \\
\hline All-cause emergency department visits & $157285(84.8)$ & $7.7 \pm 13.4 ; 4(2-9)$ & $3.1 \pm 5.2$ & $3.4 \pm 5.6$ & $3.7 \pm 6.1$ & $3.7 \pm 5.5$ \\
\hline All-cause hospital admission & $100052(53.9)$ & $3.0 \pm 3.3 ; 2(1-4)$ & $1.8 \pm 1.6$ & $1.9 \pm 1.6$ & $1.9 \pm 1.7$ & $2.0 \pm 1.8$ \\
\hline ICU admission & $29453(15.9)$ & $1.8 \pm 1.6 ; 1(1-2)$ & $1.5 \pm 1.1$ & $1.5 \pm 1.0$ & $1.5 \pm 1.1$ & $1.5 \pm 1.1$ \\
\hline
\end{tabular}

Data are presented as $\mathrm{n}(\%)$, mean \pm SD or median (interquartile range) during cohort follow-up. ICU: intensive care unit. ${ }^{\#}$ : calculated for individuals in the cohort requiring these services within each 3-year time period. 
TABLE 3 Diagnosis of patients receiving home respiratory support

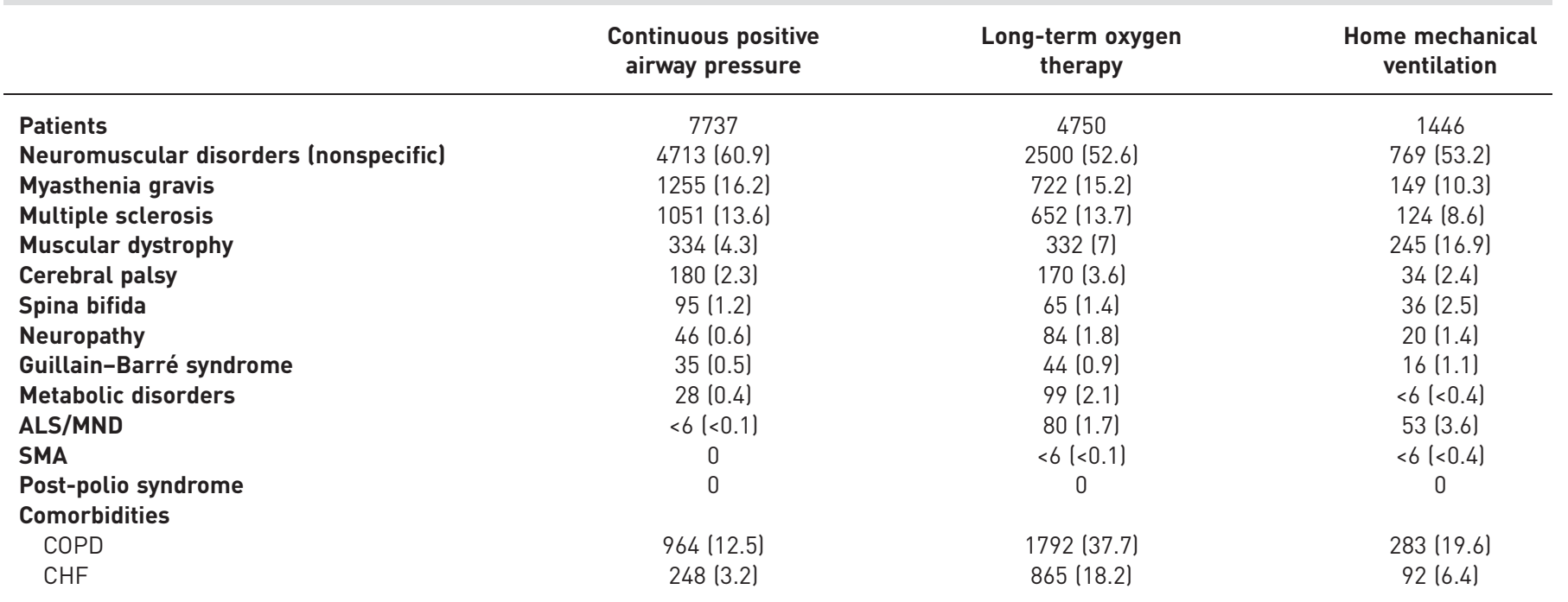

Data are presented as $\mathrm{n}$ or $\mathrm{n}(\%)$. ALS: amyotrophic lateral sclerosis; MND: motor neurone disease; SMA: spinal muscular atrophy; COPD: chronic obstructive pulmonary disease; CHF: congestive heart failure.

(nonrespiratory) consultation (table 2). The most commonly consulted nonrespiratory specialists in the outpatient setting were surgeons (46760 (25\%)), family medicine $(28780(16 \%))$ and internal medicine (16852 (9\%)); only 18388 (10\%) individuals were seen by a neurologist. Admission to a rehabilitation facility occurred for 13671 (7.4\%) individuals with a median (IQR) length of stay of 28 (14-51) days. Admission to a long-term care facility occurred for 9042 (4.9\%) individuals. Of the cohort, 70033 (38\%) NMD individuals received home care services for a median (IQR) of 32 (9-195) h overall in the year services were approved. The most common services were from registered nurses (23831 (13\%)), case managers (11914 (6\%)) and combined personal support/homemaker services (10178 (5\%)). 34336 (18.5\%) individuals died during the follow-up period.

\section{Variables associated with emergency department presentation or hospital admission}

Individuals with ALS/MND had more emergency department visits compared with other types of NMD. Those with variably progressive disease had more hospital admissions $(p<0.0001)$. Individuals with spina bifida, cerebral palsy or multiple sclerosis had the fewest hospital admissions $(\mathrm{p}<0.0001)$. Those in the oldest age category had more emergency department visits and the most hospital admissions (all $\mathrm{p}<0.0001$ ). Males, individuals in the poorest income quintile, Canadian citizens (compared with immigrants or refugees), those living in rural locations and those requiring assistive ventilation or other medical technology had more emergency department visits and hospital admissions (all $\mathrm{p}<0.0001$ ). Individuals with comorbidity had more emergency department visits and hospital admissions, with those with CHF having the most emergency department visits and those with cardiomyopathy the most hospital admissions (table 4).

\section{Health service utilisation before and after ventilation}

For the subset of 552 individuals with home ventilator approval and healthcare utilisation data available in the 3 years before and 3 years after approval, we found an increase in the mean \pm sD number of respiratory specialist outpatient visits per patient from $8.9 \pm 21.7$ to $22.7 \pm 44.7$ visits in 3 years $(\mathrm{p}<0.0001)$. Frequency of PFTs also increased, whereas frequency of sleep studies decreased (table 5). We found no difference in frequency of respiratory-related or all-cause emergency department visits, hospital or ICU admissions, or length of emergency department visit or hospital length of stay. Mean ICU length of stay decreased from 43.7 to 27.3 days $(\mathrm{p}=0.04)$.

\section{Discussion}

In this retrospective longitudinal population-based study of adults with NMD, we found the burden of acute healthcare utilisation associated with respiratory complications was moderate with $22 \%$ visiting the emergency department, on average 1.6 times every 3 years, and 8\% requiring hospital admission, on average 1.4 times every 3 years. Interestingly, of those admitted to hospital for respiratory complications, 
TABLE 4 Variables associated with the number of emergency department visits and hospital admissions after neuromuscular disease (NMD) diagnosis

\begin{tabular}{|c|c|c|c|c|}
\hline & \multicolumn{2}{|c|}{ Emergency department visits } & \multicolumn{2}{|c|}{ Hospital admissions } \\
\hline & $\begin{array}{l}\text { Incidence rate } \\
\text { ratio }(95 \% \text { CI) }\end{array}$ & p-value & $\begin{array}{l}\text { Incidence rate } \\
\text { ratio }(95 \% \mathrm{CI})\end{array}$ & p-value \\
\hline \multicolumn{5}{|l|}{ NMD ${ }^{\#}$} \\
\hline Slowly or nonprogressive & Reference & & Reference & \\
\hline Rapidly progressive & $4.36(3.89-4.88)$ & $<0.0001$ & $1.27(0.98-1.65)$ & 0.0677 \\
\hline Variably progressive & $1.12(1.1-1.13)$ & $<0.0001$ & $1.22(1.17-1.27)$ & $<0.0001$ \\
\hline Other & $1.18(1.16-1.2)$ & $<0.0001$ & $0.84(0.8-0.88)$ & $<0.0001$ \\
\hline \multicolumn{5}{|l|}{ Age group } \\
\hline $18-39$ years & Reference & & Reference & \\
\hline 40-64 years & $0.81(0.8-0.83)$ & $<0.0001$ & $1.06(1.02-1.1)$ & 0.0044 \\
\hline$\geq 65$ years & $1.03(1.01-1.05)$ & 0.0021 & $2.17(2.08-2.27)$ & $<0.0001$ \\
\hline \multicolumn{5}{|l|}{ Sex } \\
\hline Male & Reference & & Reference & \\
\hline Female & $0.89(0.87-0.9)$ & $<0.0001$ & $0.89(0.86-0.91)$ & $<0.0001$ \\
\hline \multicolumn{5}{|l|}{ Neighbourhood income quintile } \\
\hline 1 (poorest/lowest) & Reference & & Reference & \\
\hline 2 & $0.83(0.82-0.85)$ & $<0.0001$ & $0.86(0.82-0.9)$ & $<0.0001$ \\
\hline 3 & $0.76(0.74-0.77)$ & $<0.0001$ & $0.83(0.79-0.87)$ & $<0.0001$ \\
\hline 4 & $0.7(0.68-0.71)$ & $<0.0001$ & $0.79(0.76-0.83)$ & $<0.0001$ \\
\hline 5 (richest/highest) & $0.64(0.63-0.65)$ & $<0.0001$ & $0.72(0.69-0.75)$ & $<0.0001$ \\
\hline \multicolumn{5}{|l|}{ Rural residence } \\
\hline No & Reference & & Reference & \\
\hline Yes & $1.92(1.89-1.96)$ & $<0.0001$ & $1.19(1.13-1.24)$ & $<0.0001$ \\
\hline \multicolumn{5}{|l|}{ Immigration status } \\
\hline Citizen & Reference & & Reference & \\
\hline Immigrant & $0.63(0.61-0.64)$ & $<0.0001$ & $0.83(0.78-0.88)$ & $<0.0001$ \\
\hline Refugee & $0.76(0.72-0.8)$ & $<0.0001$ & $0.77(0.68-0.86)$ & $<0.0001$ \\
\hline \multicolumn{5}{|l|}{ Ventilation status } \\
\hline None & Reference & & Reference & \\
\hline Invasive ventilation & $2.34(1.82-3.01)$ & $<0.0001$ & $2.83(1.65-4.87)$ & 0.0002 \\
\hline Noninvasive ventilation & $1.69(1.51-1.88)$ & $<0.0001$ & $1.47(1.14-1.91)$ & 0.003 \\
\hline Use of other medical technology" & $1.82(1.68-1.98)$ & $<0.0001$ & $3.51(2.98-4.12)$ & $<0.0001$ \\
\hline \multicolumn{5}{|l|}{ Comorbidities } \\
\hline COPD & $1.41(1.38-1.44)$ & $<0.0001$ & $1.52(1.45-1.58)$ & $<0.0001$ \\
\hline $\mathrm{CHF}$ & $1.71(1.66-1.77)$ & $<0.0001$ & $1.76(1.65-1.88)$ & $<0.0001$ \\
\hline Asthma & $1.3(1.28-1.33)$ & $<0.0001$ & $1.24(1.19-1.29)$ & $<0.0001$ \\
\hline Cardiomyopathy & $1.33(1.14-1.56)$ & 0.0004 & $2.18(1.59-3)$ & $<0.0001$ \\
\hline Arrhythmias & $1.45(1.38-1.53)$ & $<0.0001$ & $1.87(1.69-2.08)$ & $<0.0001$ \\
\hline \multicolumn{5}{|l|}{ CADGs } \\
\hline Few $(n=1-7)$ & Reference & & Reference & \\
\hline Many $(n=8-16)$ & $1.74(1.72-1.76)$ & $<0.0001$ & $1.46(1.42-1.51)$ & $<0.0001$ \\
\hline None & $0.84(0.79-0.89)$ & $<0.0001$ & $1.1(0.95-1.26)$ & 0.2092 \\
\hline
\end{tabular}

only $9 \%$ had an inpatient respiratory specialist consultation. One-third of our cohort saw respiratory specialists in an outpatient setting on average every 9 months and underwent PFT monitoring on average every 18 months. In comparison, based on health administration database documentation of physician speciality, a neurologist saw only $10 \%$ of our cohort of adults with NMD. Approximately $6 \%$ of the cohort experienced substantial respiratory comorbidity requiring LTOT, CPAP or HMV. Approval for HMV was accompanied by an increase in respiratory specialist outpatient visits and assessment of PFTs in the subsequent 3 years, but did not affect the frequency of emergency department visits or hospitalisation.

In this real-world study, for those individuals who received specialist respiratory care, on average frequency of access to respiratory specialists, PFT and PSG monitoring reflects current guideline recommendations, although we found considerable variability particularly in terms of frequency of clinic visits. The American 
TABLE 5 Healthcare utilisation before and after initiation of home mechanical ventilation (HMV) in 552 individuals

\begin{tabular}{|c|c|c|c|c|c|c|c|c|}
\hline & Individuals" & $\begin{array}{l}\text { Overall } \\
\text { prior }^{\natural 1}\end{array}$ & $\begin{array}{l}\text { Overall } \\
\text { after }^{+}\end{array}$ & p-value & Individuals ${ }^{\S}$ & $\begin{array}{l}1 \text { year } \\
\text { prior }\end{array}$ & $\begin{array}{l}1 \text { year } \\
\text { after }\end{array}$ & p-value \\
\hline $\begin{array}{l}\text { Respiratory-related emergency } \\
\text { department visits }\end{array}$ & $196(36)$ & $1.7 \pm 1.9$ & $1.7 \pm 1.8$ & 0.95 & $136(27)$ & $1.4 \pm 1.3$ & $1.4 \pm 1.0$ & 0.72 \\
\hline Length of emergency department visit $h$ & & $17.0 \pm 31.8$ & $16.9 \pm 23.6$ & 0.97 & & $18.9 \pm 32.6$ & $14.5 \pm 21.4$ & 0.32 \\
\hline Respiratory-related hospital admission & 162 (29) & $1.7 \pm 1.4$ & $1.6 \pm 1.4$ & 0.62 & 132 (27) & $1.5 \pm 1.2$ & $1.4 \pm 1.0$ & 0.70 \\
\hline Length of hospital stay days & & $30.8 \pm 42.9$ & $22.9 \pm 36.9$ & 0.17 & & $30.5 \pm 43.4$ & $24.2 \pm 43.0$ & 0.38 \\
\hline Pulmonary function tests & $465(84)$ & $3.1 \pm 3.0$ & $4.1 \pm 7$ & 0.02 & $448(90)$ & $2.3 \pm 1.7$ & $2.8 \pm 5.1$ & 0.10 \\
\hline Sleep studies & $251(51)$ & $1.6 \pm 0.9$ & $1.3 \pm 0.6$ & $<0.0001$ & $229(46)$ & $1.4 \pm 0.6$ & $1.1 \pm 0.3$ & $<0.0001$ \\
\hline All-cause emergency department visits & $464(45)$ & $3.9 \pm 4.5$ & $3.7 \pm 4.9$ & 0.53 & $407(82)$ & $2.4 \pm 2.5$ & $2.4 \pm 2.5$ & 0.81 \\
\hline Length of emergency department visit $h$ & & $25.4 \pm 35.4$ & $30.9 \pm 44.3$ & 0.06 & & $19.8 \pm 27.6$ & $19.7 \pm 25.6$ & 0.93 \\
\hline All-cause hospital admission & $406(74)$ & $2.2 \pm 1.8$ & $2.2 \pm 2.0$ & 0.77 & $347(70)$ & $1.7 \pm 1.2$ & $1.7 \pm 1.2$ & 0.52 \\
\hline Length of hospital stay days & & $39.0 \pm 81.2$ & $35.4 \pm 75.7$ & 0.57 & & $37.7 \pm 80.7$ & $33.0 \pm 81.1$ & 0.59 \\
\hline
\end{tabular}

Data are presented as $\mathrm{n}(\%)$ or mean $\pm \mathrm{SD}$, unless otherwise stated. ICU: intensive care unit. Amyotrophic lateral sclerosis/motor neurone disease $n=23$, cerebral palsy $n=20$, Guillain-Barré syndrome $n<6$, metabolic disorders $n=7$, multiple sclerosis $n=15$, muscular dystrophy $n=52$, myasthenia gravis $n=59$, neuromuscular disorders (nonspecific) $n=355$, neuropathy $n=7$, post-polio syndrome $n<6$, spina bifida $n=6$ and $s p i n a l$ muscular atrophy $\mathrm{n}<6$. \#: individuals that utilised these healthcare services in both the 3 years preceding and 3 years following approval for

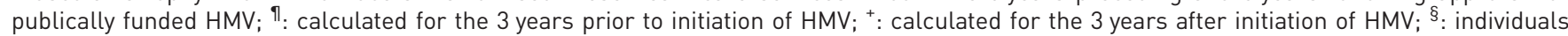
that utilised these healthcare services 1 year before and 1 year after initiation of HMV.

Thoracic Society $[12,35]$ and Centers for Disease Control and Prevention guidelines $[9,13]$ recommend monitoring and treatment of respiratory complications should be performed by respiratory specialists with clinic visits every 3-12 months depending on disease progress. Similarly, evaluation of PFTs is recommended at baseline and with increasing frequency for those with progressive disease. Reasons for wide variation in visit frequency may be attributed to the variable disease progression in NMD, but may also reflect variability in access to specialist respiratory care. Comparison with international practice is challenging as data are limited and are not reported at a population level. In a US database describing respiratory care over 11 years for 208 males with Duchenne muscular dystrophy, a group with progressive disease and generally adherent with therapy with a good response to respiratory interventions, fewer than $32 \%$ were evaluated by a respiratory specialist in $2010-2011$ and no more than $50 \%$ had PFTs measured twice yearly [8]. In a 2011-2012 cross-sectional European survey, again of Duchenne muscular dystrophy reporting on 1062 individuals, pulmonary function was not assessed regularly in $71 \%$ of nonambulatory patients [36]. As described later in this section, we were unable to isolate Duchenne muscular dystrophy within health administrative databases to enable comparison.

Our data demonstrate the considerable burden of NMD on the public healthcare system. All-cause public health service utilisation for individuals with NMD was substantial with $85 \%$ visiting the emergency department on average more than once a year, equivalent to over 1.2 million visits in 12 years, over $50 \%$ requiring hospitalisation on average twice every 3 years, and $16 \%$ requiring ICU admission, twice on average during cohort follow-up. After adjusting for multiple factors, patients with ALS/MND were the highest users of the emergency department among our cohort. Reasons for high use of emergent care likely include lack of access to speciality care, lack of care integration, lack of respite and inexperience of general practitioners with this disease [37, 38]. From our data, we can conclude that strategies are needed urgently to improve community and social supports for all patients with NMD that minimise emergent healthcare utilisation, but particularly those with ALS/MND.

Provision of publicly funded respiratory devices and community respiratory therapy was low considering the level of respiratory morbidity of the cohort. In Ontario, although all approvals for publicly funded noninvasive and invasive ventilators are captured in the ADP database, other respiratory equipment including CPAP and bilevel devices may be obtained from nonpublicly funded sources or purchased by individuals [39], and therefore our data may underestimate the use of respiratory support for NMD. Furthermore, availability of respiratory therapists in the community is extremely limited in Ontario [40]. Interestingly, despite a considerable increase in the frequency of PFT monitoring and respiratory specialist 
review after HMV commencement, there was no difference in the need for emergent care or hospital admission. Reasons for this might reflect ongoing disease progression or may relate to the limited availability of community healthcare workers for ventilator-assisted individuals [41].

A major strength of our study that provides unique data on the respiratory management of NMD is the use of real-world data from health administrative databases to capture health utilisation on a population-based level. Our study has limitations. We cannot rule out misclassification, or under- or over-ascertainment, particularly using physician billing codes to identify NMD. The nonspecific neuromuscular category comprised individuals identified with the OHIP billing code 349 that likely included individuals with ALS/MND and muscular dystrophy for whom we were unable to further delineate diagnosis through an ICD code. Limitations of health administrative database coding prevented more disease-specific description of healthcare utilisation (i.e. Duchenne muscular dystrophy, the most common muscular dystrophy) as well as an accurate estimation of disease progression on an individual patient level or within disease categories. We were unable to account for other potential confounders of emergency department presentation or hospital admission such as patient mobility status and the primary language spoken as these data are not available in health administrative databases. Additionally, health administrative databases do not enable us to assess if all individuals with NMD that should have received specialist respiratory care did receive this care. Lastly, we were unable to assess use of airway clearance strategies as mechanical insufflation/exsufflation devices were publicly funded only from 2014 onwards (prior to this date devices were covered by third-party insurers or personal expense).

\section{Conclusions}

In this heterogeneous population of adults with $\mathrm{NMD}$, we found approximately one-fifth required emergent care for respiratory complications and one-third received ongoing respiratory specialist care. For those receiving this care, frequency of monitoring adhered to guideline recommendations; however, substantial variation was noted, which likely reflects variable disease progression but also variable access to specialist care. Emergent healthcare utilisation was substantial, emphasising the burden of NMD on the healthcare system and the urgent need to improve community and social supports, particularly for ALS/ MND patients.

Acknowledgements: This study was supported by the Institute for Clinical Evaluative Sciences (ICES), which is funded by an annual grant from the Ontario Ministry of Health and Long-Term Care (MOHLTC). The opinions, results and conclusions reported in this paper are those of the authors and are independent from the funding sources. No endorsement by ICES or the Ontario MOHLTC is intended or should be inferred. Parts of this material are based on data and information compiled and provided by the Canadian Institute for Health Information (CIHI). However, the analyses, conclusions, opinions and statements expressed herein are those of the author, and not necessarily those of CIHI. Permission was received to use the Immigration, Refugees and Citizenship Canada's Permanent Resident Database to characterise immigration status and the Johns Hopkins ACG system for describing Collapsed Aggregated Diagnosis Groups.

Author contributions: L. Rose, D. McKim, R. Amin and S. Katz conceived of the study; all authors were involved in design of the study; L. Rose, and A. Gershon were involved in cohort creation; all authors contributed to analysis and interpretation of the data and writing the article before submission.

Conflict of interest: None declared.

Support statement: The study was funded by a Respiratory Care grant from Muscular Dystrophy Canada. L. Rose holds a Canadian Institutes of Health Research (CIHR) New Investigator Awards. R. Goldstein holds the National Sanitarium Association Chair in Respiratory Rehabilitation Research. A. Gershon holds a CIHR Foundation Grant and received funding from a Physicians Services Incorporated Fellowship for Translational Research while working on this study. Funding information for this article has been deposited with the Crossref Funder Registry.

\section{References}

$1 \quad$ Hill N. Ventilator management for neuromuscular disease. Semin Respir Crit Care Med 2002; 23: 293-305.

2 Tzeng A, Bach J. Prevention of pulmonary morbidity for patients with neuromuscular disease. Chest 2000; 118: $1390-1396$.

3 Panitch $\mathrm{H}$. The pathophysiology of respiratory impairment in pediatric neuromuscular diseases. Pediatrics 2009; 123: Suppl. 4, S215-S218.

4 Gomez-Merino E, Bach J. Duchenne muscular dystrophy: prolongation of life by noninvasive ventilation and mechanically assisted coughing. Am J Phys Med Rehabil 2002; 81: 411-415.

5 McKim D, Katz S, Barrowman N, et al. Lung volume recruitment slows pulmonary function decline in Duchenne muscular dystrophy. Arch Phys Med Rehabil 2012; 93: 1117-1122.

6 Howard R. Respiratory failure because of neuromuscular disease. Curr Opin Neurol 2016; 29: 592-601.

7 Hull J, Aniapravan R, Chan E, et al. British Thoracic Society guideline for respiratory management of children with neuromuscular weakness. Thorax 2012: 67: 1-40.

8 Andrews J, Soim A, Pandya S, et al. Respiratory care received by individuals with Duchenne muscular dystrophy from 2000 to 2011. Respir Care 2016: 61: 1349-1359. 
9 Bushby K, Finkel R, Birnkrant D, et al. Diagnosis and management of Duchenne muscular dystrophy, part 2: implementation of multidisciplinary care. Lancet Neurol 2010: 9: 177-189.

10 Bach JR. Respiratory muscle aids to avert respiratory failure and tracheostomy: a new patient management paradigm. Can J Respir Ther 2010: 46: 24-32.

11 Bach JR, Martinez D. Duchenne muscular dystrophy: continuous noninvasive ventilatory support prolongs survival. Respir Care 2011; 56: 744-750.

12 Finder J, Birnkrant D, Carl J, et al. Respiratory care of the patient with Duchenne muscular dystrophy: ATS consensus statement. Am J Respir Crit Care Med 2004; 170: 456-465.

13 Birnkrant D, Bushby K, Amin R, et al. The respiratory management of patients with Duchenne muscular dystrophy: a DMD Care Considerations Working Group specialty article. Pediatr Pulmonol 2010; 45: 739-748.

14 Wang C, Bonnemann C, Rutkowski A, et al. Consensus statement on standard of care for congenital muscular dystrophies. J Child Neurol 2010; 25: 1559-1581.

15 Agency for Clinical Innovation Respiratory Network. Domiciliary Non-invasive Ventilation in Adult Patients: A Consensus Statement. Chatswood, Agency for Clinical Innovation, 2012.

16 Miller R, Jackson C, Kasarskis E, et al. Practice parameter update: the care of the patient with amyotrophic lateral sclerosis: drug, nutritional, and respiratory therapies (an evidence-based review): report of the Quality Standards Subcommittee of the American Academy of Neurology. Neurology 2009; 73: 1218-1226.

17 McKim D, Road J, Avendano M, et al. Home mechanical ventilation: a Canadian Thoracic Society clinical practice guideline. Can Respir J 2011; 18: 197-215.

18 Windisch W, Geiseler J, Simon K, et al. German national guideline for treating chronic respiratory failure with invasive and non-invasive ventilation: revised edition 2017 - part 1. Respiration 2018; 96: 66-97.

19 Windisch W, Geiseler J, Simon K, et al. German national guideline for treating chronic respiratory failure with invasive and non-invasive ventilation - revised edition 2017: part 2. Respiration 2018; 96: 171-203.

20 Katz S, McKim D, Hoey L, et al. Respiratory management strategies for Duchenne muscular dystrophy: practice variation amongst Canadian sub-specialists. Pediatr Pulmonol 2013: 48: 59-66.

21 Hannan L, Sahi H, Road J, et al. Care practices and health-related quality of life for individuals receiving assisted ventilation. A cross-national study. Ann Am Thorac Soc 2016; 13: 894-903.

22 Statistics Canada. 2011 Census profile. 2013. www12.statcan.gc.ca/census-recensement/index-eng.cfm Date last accessed: November 28, 2017.

23 Herring A, Ginde A, Fahimi J, et al. Increasing critical care admissions from U.S. emergency departments, 20012009. Crit Care Med 2013: 41: 1197-1204.

24 Stephenson A, Hux J, Tullis E, et al. Socioeconomic status and risk of hospitalization among individuals with cystic fibrosis in Ontario, Canada. Pediatr Pulmonol 2011: 46: 376-384.

25 Juurlink D, Preyra C, Croxford R, et al. Canadian Institute for Health Information Discharge Abstract Database: A Validation Study. Toronto, Institute for Clinical Evaluative Sciences, 2006.

26 Ray J, Redelmeier D, Urquia M, et al. Risk of cerebral palsy among the offspring of immigrants. PLoS One 2014; 9: e102275.

27 Breiner A, Widdifield J, Katzberg H, et al. Epidemiology of myasthenia gravis in Ontario, Canada. Neuromuscul Disord 2016: 26: 41-46.

28 Widdifield J, Ivers N, Young J, et al. Development and validation of an administrative data algorithm to estimate the disease burden and epidemiology of multiple sclerosis in Ontario, Canada. Mult Scler 2015; 21: 1045-1054.

29 Kwong J, Vasa P, Campitelli M, et al. Risk of Guillain-Barré syndrome after seasonal influenza vaccination and influenza health-care encounters: a self-controlled study. Lancet Infect Dis 2013; 13: 769-776.

30 Shneerson J, Simonds A. Noninvasive ventilation for chest wall and neuromuscular disorders. Eur Respir J 2002; 20: 480-487.

31 Gershon A, Wang C, Guan J, et al. Identifying patients with physician-diagnosed asthma in health administrative databases. Can Respir J 2009; 16: 183-188.

32 Gershon A, Wang C, Guan J, et al. Identifying individuals with physician diagnosed COPD in health administrative databases. COPD 2009; 6: 388-394.

33 Schultz S, Rothwell D, Chen Z, et al. Identifying cases of congestive heart failure from administrative data: a validation study using primary care patient records. Chronic Dis Inj Can 2013; 33: 160-166.

34 Austin P, van Walraven C, Wodchis W, et al. Using the Johns Hopkins Aggregated Diagnosis Groups (ADGs) to predict mortality in a general adult population cohort in Ontario, Canada. Med Care 2011; 49: 932-939.

35 Finder J. A 2009 perspective on the 2004 American Thoracic Society statement, "Respiratory Care of the Patient with Duchenne Muscular Dystrophy". Pediatrics 2009: 123: S239-S241.

36 Vry J, Gramsch K, Rodger S, et al. European cross-sectional survey of current care practices for Duchenne muscular dystrophy reveals regional and age-dependent differences. J Neuromuscul Dis 2016; 3: 517-527.

37 Hobson E, Baird W, Partridge R, et al. The TiM system: developing a novel telehealth service to improve access to specialist care in motor neurone disease using user-centered design. Amyotroph Lateral Scler Frontotemporal Degener 2018: 19: 351-361.

38 Peters M, Fitzpatrick R, Doll H, et al. Patients' experiences of health and social care in long-term neurological conditions in England: a cross-sectional survey. J Health Serv Res Policy 2013: 18: 28-33.

39 Rose L, McKim D, Katz S, et al. Home mechanical ventilation in Canada: a national survey. Respir Care 2015; 60: 695-704.

40 Leasa D, Elson S. Building a comprehensive system of services to support adults living with long-term mechanical ventilation. Can Respir J 2016: 2016: 3185389.

41 Dale C, King J, Nonoyama M, et al. Transitions to home mechanical ventilation: the experiences of Canadian ventilator-assisted adults and their family caregivers. Ann Am Thorac Soc 2018; 15: 357-364. 